\title{
Transcription factor EGR-1 transactivates the MMP1 gene promoter in response to $\mathrm{TNF} \alpha$ in $\mathrm{HaCaT}$ keratinocytes
}

\author{
Hyunjin Yeo ${ }^{1}$, Jeong Yeon Lee ${ }^{1}$, JuHwan Kim ${ }^{1}$, Sung Shin Ahn ${ }^{1}$, Jeong You Jeong ${ }^{2}$, Ji Hye Choi ${ }^{1}$, Young Han Lee ${ }^{1,2}$ E \\ Soon Young Shin $1,2, *$ \\ ${ }^{1}$ Department of Biological Sciences, Sanghuh College of Lifesciences, Konkuk University, Seoul 05029, ${ }^{2}$ Cancer and Metabolism Institute, \\ Konkuk University, Seoul 05029, Korea
}

Matrix metalloproteinase 1 (MMP-1), a calcium-dependent zinccontaining collagenase, is involved in the initial degradation of native fibrillar collagen. Tissue necrosis factor-alpha (TNF $\alpha$ ) is a pro-inflammatory cytokine that is rapidly produced by dermal fibroblasts, monocytes/macrophages, and keratinocytes and regulates inflammation and damaged-tissue remodeling. MMP-1 is induced by TNF $\alpha$ and plays a critical role in tissue remodeling and skin aging processes. However, the regulation of the MMP1 gene by TNF $\alpha$ is not fully understood. We aimed to find additional cis-acting elements involved in the regulation of TNFo-induced MMP1 gene transcription in addition to the nuclear factor-kappa B (NF-kB) and activator protein 1 (AP1) sites. Assessments of the 5'-regulatory region of the MMP1 gene, using a series of deletion constructs, revealed the requirement of the early growth response protein 1 (EGR-1)-binding sequence (EBS) in the proximal region for proper transcription by TNF $\alpha$. Ectopic expression of EGR-1, a zinc-finger transcription factor that binds to G-C rich sequences, stimulated MMP1 promoter activity. The silencing of EGR-1 by RNA interference reduced TNF $\alpha$-induced MMP-1 expression. EGR-1 directly binds to the proximal region and transactivates the MMP1 gene promoter. Mutation of the EBS within the MMP1 promoter abolished EGR-1-mediated MMP-1 promoter activation. These data suggest that EGR-1 is required for TNF $\alpha$-induced MMP1 transcriptional activation. In addition, we found that all three MAPKs, ERK1/2, JNK, and p38 kinase, mediate TNF $\alpha$-induced MMP-1 expression via EGR-1 upregulation. These results suggest that EGR-1 may represent a good target for the development of pharmaceutical agents to reduce inflammation-induced MMP-1 expression. [BMB Reports 2020; 53(6): 323-328]

*Corresponding author. Tel: +82-2-2030-7946; Fax: +82-2-34379781; E-mail: shinsy@konkuk.ac.kr

https://doi.org/10.5483/BMBRep.2020.53.6.290

Received 21 November 2019, Revised 23 December 2019, Accepted 6 January 2020

Keywords: EGR-1, EGR-1-binding sequence, Gene promoter, Mitogen-activated protein kinase, MMP1, TNF $\alpha$

\section{INTRODUCTION}

The skin is the most extensive tissue of the body and consists of three layers (epidermis, dermis, and subcutaneous tissue). The epidermis is a stratified squamous epithelium of the outermost layer, which provides a mechanical barrier against invasion of foreign substances and loss of body water. The epidermis mechanically maintains a skin structure against environmental stresses, including mechanical injury, UV radiation, and infection. Maintaining a constant skin structure requires dynamically coordinated degradation and synthesis of extracellular matrix (ECM) components.

Matrix metalloproteinases (MMPs) are a family of endopeptidases responsible for the proteolytic degradation of structural components of ECM. MMPs are associated with various physiological and pathological cellular functions, including cell migration, wound healing, angiogenesis, immune responses, cell growth and differentiation, apoptosis, and skin aging (1, 2). To date, at least 24 different MMPs have been identified in humans (1) and classified into four groups based on their domain organization; archetypal MMPs, gelatinases, matrilysins, and furin-activatable MMPs (3). Of archetypal MMPs, MMP-1 is a sub-group of collagenase involved in the initial cleavage of native fibrillar collagens (3). It functions in tissue remodeling, inflammation, and skin photoaging (4) and has been implicated in various pathological processes, such as tumor cell invasion, arthritis, and atherosclerosis (5). Like other MMPs, MMP-1 is expressed at a low level in most normal cells but rapidly induced in response to exogenous signals, including growth factors, inflammatory cytokines, and oncogenic transformation. MMP-1 expression largely depends on transcriptional activity.

Wounds and other inflammatory responses in the skin lead to tissue remodeling characterized by elevated inflammatory cytokines. Tissue necrosis factor $\alpha$ (TNF $\alpha$ ) is one of potent pro-inflammatory cytokine produced by dermal fibroblasts, monocytes/macrophages, and keratinocytes in response to ultraviolet irradiation and inflammatory signals (6). TNF $\alpha$ promotes tissue remodeling by increasing the secretion of multiple cytokines and inflammatory mediators in keratinocytes and dermal fibroblasts (6). MMP-1 is also induced by TNF $\alpha$ and contributes to the remodeling of damaged tissues (4). The 
role of mitogen-activated protein kinases (MAPKs), including extracellular signal-regulated kinase (ERK), c-Jun-N-terminal kinase (JNK), and p38 kinase, is well established in the regulation of TNF $\alpha$-induced MMP-1 expression via AP1 upregulation (3).

Early Growth Response (EGR)- 1 is an inducible zinc-finger transcription factor that binds to GC-rich sequences (7). Generally, EGR-1 is expressed at the base level but is rapidly and transiently induced by various growth factors and inflammatory cytokines in a variety of cell types $(8,9)$. In turn, activated EGR-1 regulates the expression of many inflammation-related genes (10-12). Indeed, EGR-1 regulates interleukin (IL)-13-induced lung inflammation by expressing $\mathrm{CC}$ and $\mathrm{CXC}$ chemokines (13) and TNF $\alpha$-induced CXCL1 expression (8). Furthermore, EGR-1 induces many pro-inflammatory cytokines in keratinocytes during skin inflammation; TNF $\alpha$ autoregulation (14), IL-17A-induced psorian expression (15), and IL-33-induced thymic stromal lymphopoietin (TSLP) expression (16). We previously showed that EGR-1 transactivates the MMP9 gene in response to TNF $\alpha$ stimulation in HeLa cells (17). Although EGR-1 is induced by TNF $\alpha$ through MAPK pathways in diverse cell types $(8,17,18)$, the functional role of EGR-1 in the regulation of the MMP1 gene promoter is not characterized.

We aimed to investigate the role of EGR-1 in the regulation of MMP-1 expression. The objective of this study is to identify the EGR-1-binding sites in the 5'-regulatory region of the $M M P-1$ gene and to determine the molecular mechanisms involved in TNF $\alpha$-induced MMP-1 expression in HaCaT keratinocyte model cell line. We demonstrated here that the EGR1-binding motif is a positive cis-acting element necessary for the full-activation of TNFo-induced MMP-1 expression. Also, we showed that elevation of EGR-1 expression leads to the increase in MMP1 gene promoter activity and that the silencing of EGR-1 by RNA interference abrogates TNF $\alpha$-induced MMP-1 expression. We propose that EGR-1 is a potent transcription factor for regulating the MMP1 gene transcription.

\section{RESULTS AND DISCUSSION}

\section{EGR-1-binding cis-acting element is necessary for TNF $\alpha$-induced MMP1 promoter activity}

The 5'-regulatory region of the MMP1 gene contains multiple cis-acting elements; nuclear factor-kappa B (NF- $\mathrm{KB})$, activator protein 1 (AP1), erythroblastosis twenty-six-1 (ETS-1), serum amyloid A-activating factor 1 (SAF1), and polyoma enhancer element A-3 (PEA-3), and signal transducer and activator of transcription sites (STAT3) $(19,20)$. In addition to these regulatory elements, we sought to find a new cis-acting element involved in TNF $\alpha$-induced MMP1 transcription. To characterize the regulatory regions responsible for TNF $\alpha$ stimulation, we constructed a series of $5^{\prime}$ deletion constructs of the MMP1 promoter $(-569 /+87,-356 /+87$, and $-152 /+87)$ in the luciferase-based reporter plasmid and mapped the active region responsible for TNF $\alpha$ stimulation (Fig. 1A). Promoter reporter

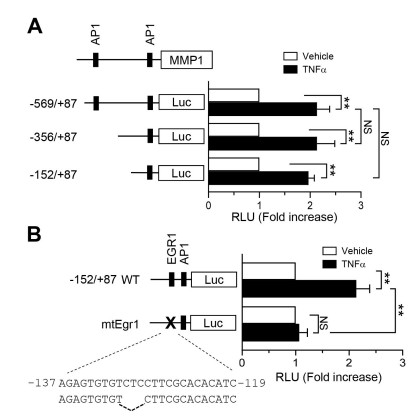

Fig. 1. The EGR-1-binding sequence in the MMP1 promoter is functional. (A) HaCaT cells were transfected with $0.1 \mu \mathrm{g}$ of a series of 5'-deletion constructs of MMP-1 promoter-reporter (pMMP1Luc). After $48 \mathrm{~h}$, the cells were treated with either vehicle (PBS) or $10 \mathrm{ng} / \mathrm{ml}$ TNF $\alpha$ and incubated for an additional $8 \mathrm{~h}$; subsequently, luciferase activities were determined. Bars represent the mean + S.D. $(n=3)$. **P $<0.001 ; N S$, not significant; by Sidak's multiple comparison test. (B) HaCaT cells were transfected with $0.1 \mu \mathrm{g}$ of wild-type (WT) pMMP1-Luc $(-152 /+87)$ or EGR-1 site mutant construct (mtEGR-1). After $48 \mathrm{~h}$, cells were treated with either vehicle (PBS) or $10 \mathrm{ng} / \mathrm{ml} \mathrm{TNF} \alpha$ for an additional 8 h; subsequently, luciferase activities were determined. Bars represent the mean \pm S.D. $(n=3)$. ${ }^{* * P}<0.001$; NS, not significant; by Sidak's multiple comparison test.

activity upon TNF $\alpha$ stimulation still occurred following the deletion of a region up to nucleotide position $-151 \mathrm{bp}$, suggesting that the proximal region between -152 and +87 contains TNF $\alpha$ responsive cis-acting regulatory elements. We analyzed the transcription factor binding sites between -152 and +87 bp using a web-based Matlnspector program (http:// www.genomatix.de/). We found a putative EGR-1-binding sequence (EBS) located at $-137 /-119$ near the AP1 site (Supplementary Fig. S1).

To determine the role of the putative EBS in TNF $\alpha$-induced MMP-1 expression, we disrupted EBS by site-specific deletion of core nucleotide (CTC). Disruption of the EGR-1-binding core sequence within the $-152 /+87$ construct (mtEBS) resulted in a near-complete loss of TNF $\alpha$-stimulated promoter-reporter activity ( $P<0.001, n=3$ ), compared to the wild-type construct (Fig. 1B), suggesting that the EGR-1-binding motif at $-137 /-119$ is necessary for TNF $\alpha$-mediated MMP1 transcription.

\section{EGR-1 binds to the MMP1 gene promoter}

Next, we assessed whether EGR-1 binds to the putative EBS motif within the proximal MMP1 promoter region. DNA binding was assessed using the electrophoretic mobility shift assay (EMSA) with Sf21 insect cell lysates overexpressing EGR-1 (Sf21/EGR-1). Biotinylated EBS oligonucleotides, but not mutated EBS (mtEBS), formed a DNA-protein complex (Fig. $2 A)$, suggesting that EGR-1 binds exclusively to the EBS motif within the proximal MMP1 promoter region. EGR-1 binding to the EBS was confirmed using the DNA-affinity precipitation assay (DAPA). Biotinylated EBS and mtEBS oligonucleotides 


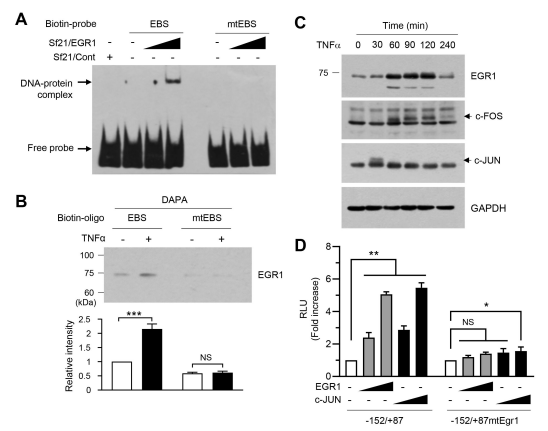

Fig. 2. EGR-1 transactivates the $M M P 1$ promoter. (A) Biotinylated EGR-1-binding sequence probe (EBS) or mutated EBS (mtEBS) was incubated with increasing concentrations of the $\mathrm{Sf} 21$ cell lysates expressing EGR-1 (Sf21/EGR-1) or WT (Sf21/Cont). Samples were separated by non-denaturing $6 \%$ acrylamide gel electrophoresis and visualized using streptavidin-conjugated horseradish peroxidase. (B) HaCaT cells were treated with $10 \mathrm{ng} / \mathrm{ml}$ TNF $\alpha$ for $1 \mathrm{~h}$. Nuclear extracts were prepared and incubated with biotinylated EBS or mtEBS for $1 \mathrm{~h}$, followed by precipitation with streptavidin-conjugated agarose beads. Immunoblotting was performed with an anti-EGR-1 antibody and analyzed by the DNA-affinity precipitation assay. (C) HaCaT cells were treated with $10 \mathrm{ng} / \mathrm{ml}$ TNF $\alpha$ for different periods (0-240 min). Cell lysates were immunoblotted with antibodies against EGR-1, c-FOS, and c-JUN. GAPDH levels were examined as an internal control. (D) HaCaT cells were co-transfected $0.1 \mu \mathrm{g}$ of wild-type $(-152 /+87)$ or EGR-1 site mutant construct $(-152 /$ +87 mtEGR-1) with 50 or $100 \mathrm{ng}$ of an expression plasmid for EGR-1 or c-JUN. After 48 h, luciferase activities were measured. Bars represent the mean $\pm \mathrm{SD}(n=3)$. Bars represent the mean \pm S.D. $(n=3)$. $* P=0.0336 ; * * P<0.001$; NS, not significant; by Sidak's multiple comparison test.

were incubated with nuclear extracts of HaCaT cells stimulated with TNF $\alpha$ or vehicle. After pull-down with streptavidin-conjugated agarose beads, oligonucleotide-binding proteins were eluted and immunoblotted using anti-EGR-1 antibodies. Similar to the EMSA result, TNF $\alpha$ stimulation increased EGR-1 binding to the EBS, but not to the mtEBS (Fig. 2B), suggesting that EGR-1 directly interacts with the proximal EBS in the MMP1 promoter region.

EGR-1 protein accumulated in a pattern similar to AP1 components (c-FOS and c-JUN) following TNF $\alpha$ stimulation in $\mathrm{HaCaT}$ cells (Fig. 2C). To investigate whether EGR-1 alone could transactivate the MMP1 promoter, we co-transfected EGR-1 expression plasmid and the $-152 /+87$ promoterreporter. The c-JUN component of AP1 was used as a positive control. Similar to c-JUN, exogenous EGR-1 expression elicited a significant DNA concentration-dependent increase in promoter activity of $-152 /+87$ but not $-152 /+87$ mtEGR- 1 (Fig. 2D), suggesting EGR-1 is a positive regulator of the MMP1 gene. Interestingly, disruption of the EBS (mtEBS) also abrogated c-JUN stimulation of promoter activity, suggesting that EGR-1 is also necessary for the AP1 trans-acting activity in MMP1 transcription. As the Fos-Jun heterodimer interacts with EGR-1 in the promoter of the mouse tyrosine hydroxylase gene
A

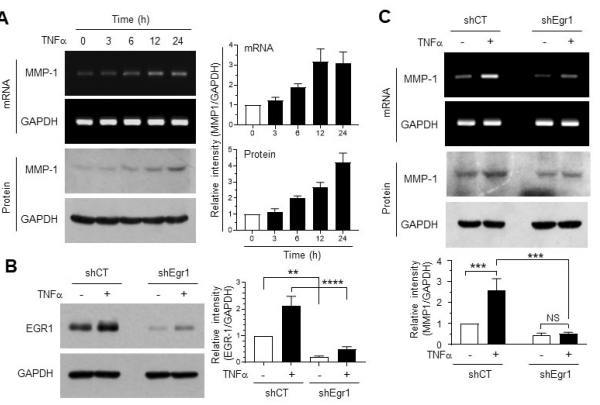

Fig. 3. Effect of EGR-1 silencing on TNF $\alpha$-induced MMP-1 expression. (A) HaCaT cells were treated with $10 \mathrm{ng} / \mathrm{ml}$ TNF $\alpha$ for various times $(0-24 \mathrm{~h})$. MMP-1 mRNA and protein levels were determined by RT-PCR and immunoblot, respectively. GAPDH levels were examined as an internal control. (B, C) HaCaT variant cells expressing scrambled (shCT) or EGR-1 shRNA (shEGR-1) were treated with $10 \mathrm{ng} / \mathrm{ml} \mathrm{TNF} \alpha$ for $1 \mathrm{~h}$ (for EGR-1) or $12 \mathrm{~h}$ (for MMP-1). GAPDH levels were examined as an internal control. The band intensities of MMP-1 relative to GAPDH were measured using Image s software. Bars represent the mean \pm S.D. $(n=3) . * * P=0.0026 ; * * * P<0.001 ; * * * P<0.0001 ; \mathrm{NS}$, not significant; by Sidak's multiple comparison test.

(21), EGR-1 may directly or indirectly interact with AP1 to enhance MMP1 promoter activity.

\section{Knockdown of EGR-1 expression reduces TNF $\alpha$-induced MMP-1 expression}

Upon TNF $\alpha$ stimulation, MMP-1 mRNA and protein levels were upregulated in a time-dependent manner in HaCaT cells (Fig. 3A). To verify the role of EGR-1 in TNF $\alpha$-induced MMP-1 expression, we established $\mathrm{HaCaT}$ variant cells expressing shRNAs against scrambled control (shCT) and EGR-1 (shEGR-1). shRNA-mediated knockdown of EGR-1 expression was confirmed by immunoblotting (Fig. 3B). EGR-1 silencing reduced both basal and TNF $\alpha$-induced MMP-1 mRNA and protein levels (Fig. 3C). Thus, EGR-1 is necessary for $\mathrm{TNF} \alpha$-induced MMP-1 expression.

\section{MAPK pathways mediate TNF $\alpha$-induced MMP-1 expression via EGR-1 expression}

MAPK pathways regulate EGR- 1 expression in various cell types $(8,17,18,22-25)$. In serum-starved HaCaT cells, ERK1/2, p38, and JNK1/2 MAPK phosphorylation rapidly increased within 15 min following TNF $\alpha$ stimulation (Fig. 4A), suggesting that TNF $\alpha$ activates three different MAPKs in HaCaT cells. To investigate the possible relationship between MAPK activation and EGR-1 upregulation, we examined the effects of pharmacological MAPK inhibitors on EGR-1 expression. MEK inhibitor U0126, JNK1/2 inhibitor SP600125, and p38 kinase inhibitor SB203580 significantly ( $<<0.001, n=3$ ) abrogated TNF $\alpha$-induced c-JUN, c-FOS, and EGR-1 protein accumulation (Fig. 4B). RT-PCR revealed considerable inhibition of MMP-1 mRNA expression by all three MAPK inhibitors (Fig. 4C). To 


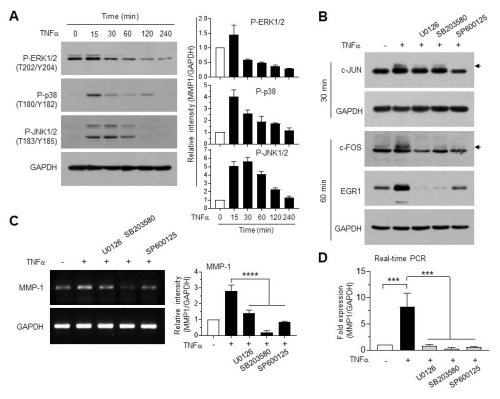

Fig. 4. Role of MAPKs in EGR-1-mediated MMP-1 expression by TNF $\alpha$. (A) HaCaT cells were treated with $10 \mathrm{ng} / \mathrm{ml}$ TNF $\alpha$ for various times (0-240 min). Immunoblotting was performed using a phospho-specific antibody against ERK1/2 (Thr202/Tyr204), p38 (Thr180/Tyr182), or JNK1/2 (Thr183/Tyr185) MAPK. GAPDH levels were examined as an internal control. (B) HaCaT cells were pre-treated with U0126 $(10 \mu \mathrm{M})$, SB203580 $(20 \mu \mathrm{M})$, or SP600125 $(20 \mu \mathrm{M})$ for $30 \mathrm{~min}$ before stimulation with $10 \mathrm{ng} / \mathrm{ml} \mathrm{TNF} \alpha$. After 30 (for c-JUN) or 60 min (for c-FOS and EGR-1) of TNF $\alpha$ treatment, immunoblotting was performed with indicated antibodies. GAPDH levels were examined as an internal control. (C and D) HaCaT cells were pre-treated with U0126 (10 $\mu \mathrm{M})$, SB203580 (20 $\mu \mathrm{M})$, or SP600125 $(20 \mu \mathrm{M})$ for $30 \mathrm{~min}$ before stimulation with $10 \mathrm{ng} / \mathrm{ml} \mathrm{TNF} \alpha$. After $12 \mathrm{~h}$ of TNF $\alpha$ treatment, RT-PCR (C) and real-time PCR (D) were performed. GAPDH levels were examined as an internal control. The band intensities were measured as relative to GAPDH using Image software. Bars represent the mean \pm S.D. $(n=3)$. $* * * P<0.0001$ by Sidak's multiple comparison test.

precisely quantify the effect of MAPK inhibition, we performed real-time PCR analysis. Upon TNF $\alpha$ stimulation, MMP-1 mRNA levels were enhanced $8.33 \pm 2.52$-fold, which was significantly reduced to $0.867 \pm 0.252-, 0.333 \pm 0.208-$, and $0.600 \pm 0.100$-fold in the presence of U0126, SB203580, and SP600125, respectively (Fig. 4D). These results suggest that all three major MAPKs are involved in TNF $\alpha$-induced MMP-1 expression via EGR-1 induction.

In summary, EGR-1 is necessary for TNF $\alpha$-induced MMP-1 expression in HaCaT keratinocytes. This study uncovered evidence of a novel EGR-1-binding cis-acting element located at $-137 /-119$, which is necessary for TNF $\alpha$-induced MMP1 transcriptional activity. Although the role of EGR-1 in skin biology in vivo - wound healing and skin aging - needs further investigation, it might represent a valuable molecular target to develop new pharmaceutical agents to reduce inflammationinduced MMP-1 expression.

\section{MATERIALS AND METHODS}

\section{Reagents}

TNFa, U0126, SB203580, and SP600125 were obtained from Calbiochem (San Diego, CA, USA). The Firefly and Renilla Dual-Glo $^{\text {TM }}$ Luciferase Assay System were obtained from Promega (Madison, WI, USA). The pRL-null plasmid encoding Renilla luciferase was also purchased from Promega.
Streptavidin-agarose was obtained from Invitrogen (Carlsbad, CA, USA). Antibodies against c-FOS, c-JUN, glyceraldehyde 3-phosphate dehydrogenase (GAPDH), and early growth response 1 (EGR-1) were purchased from Santa Cruz Biotechnology (Santa Cruz, CA, USA). Phospho-extracellular signal-activated kinase (ERK)1/2 (Thr202/Tyr204), phospho-p38 (Thr180/Tyr182), and phospho-c-jun N-terminal kinase (JNK)1/2 (Thr183/Tyr185) were obtained from Cell Signaling Technology (Danvers, MA, USA). Other chemicals were purchased from Sigma-Aldrich (St. Louis, MO, USA).

\section{Cell culture}

$\mathrm{HaCaT}$ human keratinocytes were obtained from Cell Lines Service $\mathrm{GmbH}$ (Eppelheim, Germany). The cells were maintained in Dulbecco's modified Eagle's medium (DMEM) supplemented with $10 \%$ fetal bovine serum (FBS; BioWest, Kansas City, MO, USA).

\section{Plasmids}

The pRL-null plasmid encoding Renilla luciferase was purchased from Promega. Plasmids expressing EGR-1 (pcDNA3.1/EGR-1) were described elsewhere (26). Plasmids expressing c-JUN (pcDNA3.1/Jun) were obtained from Invitrogen.

\section{Generation of serial deletion and mutant constructs of human MMP-1 gene promoter-reporter}

A human MMP1 gene promoter fragment spanning nucleotides -569 to +87 was synthesized from human genomic DNA (Promega) by PCR using the primers $5^{\prime}$-TTG AAC TCC TGG GCT CAA GT-3' (forward: - 569F) and 5'-CTG CTC CAA TAT CCC AGC TA-3' (reverse: +87R). The amplified PCR products were ligated into a T\&A vector (RBC Bioscience, New Taipei City, Taiwan). Following digestion with Kpnl and Bg/ll, the fragments into the Kpnl and Bg/II sites of the pGL4-basic vector (Promega). The fragment was ligated into the pGL4-Luc basic vector (Promega) to yield pMMP1-Luc $(-569 /+87)$. A series of deletion constructs were synthesized by PCR using the pMMP1-Luc $(-569 /+87)$ plasmid as a template. The forward primer sequences were 5'-TCA GTA CAG GTG CCG AAC AG-3' (-356F) and 5'-GGT GGT GGT ACC TGT TTA CAT GGC AGA GTG TGT C-3' (-152F). One reverse primer $(+87 \mathrm{R})$ was used to generate all deletion constructs. The amplified PCR products were ligated into the PGL4-Luc basic vector, yielding pMMP1-Luc $(-356 /+87)$ and pMMP1-Luc $(-152 /+87)$, respectively. Site-specific deletion of the EGR-1binding sequence (mtEGR-1) was performed using the QuickChange site-directed mutagenesis system (Stratagene, La Jolla, CA, USA), with the pMMP1-Luc $(-152 /+87)$ construct as a template plasmid, yielding pMMP1-Luc $(-152 /+87)$ mtEGR-1. Primer sequences used to generate deletion mutants were as follows: mtEGR-1: forward, 5'-CTT CGC ACA CAT CTT GTT TGA AGT TAA TC-3'; reverse, 5'-AAG ACA CAC TCT GCC ATG TAA ACA G-3'. PCR conditions were 2 min at $94^{\circ} \mathrm{C}$, followed by 25 cycles of denaturation at $94^{\circ} \mathrm{C}$ (30 s), 
annealing at $55^{\circ} \mathrm{C}(1 \mathrm{~min})$, and elongation at $72^{\circ} \mathrm{C}(6 \mathrm{~min})$. All reporter constructs and point mutation sites were verified by DNA sequencing at Macrogen (Seoul, Republic of Korea).

\section{Luciferase promoter-reporter assay}

Reporter luciferase activity was measured as described previously (27). In some experiments, pMMP1-Luc $(-152 /+87)$ and pMMP1-Luc $(-152 /+87)$ mtEGR-1 constructs were co-transfected with mammalian expression vectors for EGR-1 (pcDNA3.1/ EGR-1) or c-Jun (pcDNA3.1/Jun). Luminescence was measured using a Centro LB960 dual luminometer (Berthold Tech, Bad Wildbad, Germany). The relative amount of luciferase activity after normalization to the Renilla luciferase signal in the untreated cells was designated as 1 .

\section{Expression of EGR-1 in Sf21 insect cells using the baculovirus system}

IPLB-Sf21 insect cells obtained from Clontech (Mountain View, CA, USA) were maintained in Grace's insect medium (Gibco, Grand Island, NY, USA) supplemented with 10\% FBS (Hyclone, Logan, UT, USA), $100 \mathrm{U} / \mathrm{ml}$ penicillin, and 100 $\mu \mathrm{g} / \mathrm{ml}$ streptomycin at $28^{\circ} \mathrm{C}$. The coding DNA sequence of EGR-1 was obtained from the expression plasmid of EGR-1 (pcDNA3.1zeo/EGR-1) by digestion with HindIII and Bg/II and ligated into the same sites of the pOET1 Transfer Plasmid (Mirus Bio, Madison, WI, USA), yielding pOET1/EGR-1. IPLB-Sf21 cells were plated into the wells of a 6-well plate at a density of $1.5 \times 10^{6}$ cells/well and incubated for $1 \mathrm{~h}$. Each well then received $100 \mathrm{ng}$ of transfer vector (pOET/EGR-1), $100 \mathrm{ng}$ viral DNA (flashBAC ${ }^{\mathrm{TM}}$, Mirus Bio), and $1.2 \mu \mathrm{l}$ of Trans IT ${ }^{\mathbb{R}}$-Insect Transfection Reagent (Mirus Bio) in $100 \mu \mathrm{l}$ serum-free Grace's insect medium. The plates were incubated for $20 \mathrm{~min}$ after gently pipetting. The mixture was added dropwise, and the plates were incubated at $28^{\circ} \mathrm{C}$ for 4 to 5 days. The cells were dislodged from each well into the fluid by scraping and collected by centrifugation. The transfected EGR-1 level was confirmed using immunoblotting.

\section{Electrophoretic mobility shift assay (EMSA)}

Protein-DNA binding was assessed by EMSA using the LightShift Chemiluminescence EMSA kit, according to the manufacturer's instructions (ThermoFisher Scientific, Waltham, MA, USA). Biotin-labeled deoxyoligonucleotide probes corresponding to the EGR-1-binding sequence (EBS; 5'-biotinAGA GTG TGT CTC CTT CGC ACA CAT C-3') and mutated sequence (mtEBS; 5'-biotin- AGA GTG TGT CTT CGC ACA CAT C-3') were synthesized by Macrogen. Wild-type control or EGR-1-expressing IPLB-Sf21 lysates $(3 \mu \mathrm{g})$ were mixed with $50 \mathrm{fmol}$ biotin-labeled EBS and mtEBS oligonucleotide probes along with $1 \mu \mathrm{g}$ poly(dl-dC) (Amersham Pharmacia Biotech Inc., Piscataway, NJ, USA). Samples were electrophoresed in non-denaturing $6 \%$ polyacrylamide gels, followed by incubation with horseradish peroxidase (HRP)-conjugated streptavidin. EGR-1-DNA complexes were visualized using an enhanced chem- iluminescence (ECL) detection system (ThermoFisher Scientific).

\section{Immunoblot analysis}

HaCaT cells treated with PBS or $10 \mathrm{ng} / \mathrm{ml}$ TNF $\alpha$ were lysed and immunoblot analysis was performed as described previously (8). The blots were developed using LumiGLO peroxidase chemiluminescent substrate (SeraCare, Milford, MA, USA). In some experiments, the relative intensities of the MMP-1 bands were quantified and normalized by GAPDH expression using ImageJ version 1.52a software (National Institutes of Health, Bethesda, MD, USA).

\section{DNA-affinity precipitation assay (DAPA)}

$\mathrm{HaCaT}$ cells were stimulated with $10 \mathrm{ng} / \mathrm{ml}$ TNF $\alpha$ for $12 \mathrm{~h}$, and nuclear extracts $(70 \mu \mathrm{g})$ were incubated with streptavidinconjugated magnetic beads (BIONEER, Daejeon, Korea) and 5 $\mu \mathrm{g}$ of biotinylated EBS (5'-biotin-AGA GTG TGT CTC CTT CGC ACA CAT C-3') or mtEBS (5'-biotin-AGA GTG TGT CTT CGC ACA CAT C-3') for 15 min. After washing twice with PBS, the pellets were cooked with Laemmli sample buffer, and the binding proteins were resolved by $10 \%$ SDS-PAGE. Immunoblotting was performed using anti-EGR-1 antibodies.

\section{Reverse transcription-polymerase chain reaction (RT-PCR)} Isolation of total RNA and synthesis of complementary DNA (cDNA) were described elsewhere (27). The resulting cDNA was subjected to PCR analysis using gene-specific primers as follows: forward MMP-1, 5'-CAA AAT CCT GTC CAG CCC ATC G-3' and reverse MMP-1, 5'-TTC GTA AGC AGC TTC AAG CCC-3'; forward GAPDH, 5'-ACC CAC TCC TCC ACC TTT G-3' and reverse GAPDH, 5'-CTC TTG TGC TGC TGG G-3'. The PCR conditions included denaturation at $95^{\circ} \mathrm{C}$ for 30 $\mathrm{s}$, annealing at $62^{\circ} \mathrm{C}$ for $30 \mathrm{~s}$, and elongation at $72^{\circ} \mathrm{C}$ for $30 \mathrm{~s}$. The amplicons were electrophoresed on $1 \%$ agarose gels and visualized by ethidium bromide staining. In some experiments, relative intensities of MMP-1 PCR bands were quantified and expressed as a ratio to GAPDH intensities using ImageJ version 1.52a software.

\section{Knockdown of EGR-1 by using shRNA}

$\mathrm{HaCaT}$ cells were incubated with lentiviral short hairpin (sh)RNA (TRCN 0000273850; MISSION ${ }^{\mathbb{R}}$ shRNA; Sigma-Aldrich) targeting EGR-1, according to the manufacturer's instructions. Two weeks after transfection, cells were collected, and the stable knockdown of EGR-1 expression was analyzed by immunoblotting.

\section{Statistical analyses}

Data were analyzed by ANOVA, followed by Sidak's or Dunnett's multiple comparisons test, using GraphPad Prism version 8.2.0 software (GraphPad Software Inc., La Jolla, CA, USA). P-values less than 0.05 were considered statistically significant. 


\section{ACKNOWLEDGEMENTS}

This study was supported by the National Research Foundation of Korea (NRF), funded by the Korea government (MSIT) (No. 2018R1A2B2004653). The paper was supported by the KU Research Professor Program of Konkuk University and by the Konkuk University Researcher Fund in 2018. We would like to thank Editage (www.editage.co.kr) for English language editing.

\section{CONFLICTS OF INTEREST}

The authors have no conflicting interests.

\section{REFERENCES}

1. Loffek S, Schilling O and Franzke CW (2011) Series "matrix metalloproteinases in lung health and disease": Biological role of matrix metalloproteinases: a critical balance. Eur Respir J 38, 191-208

2. Panwar $P$, Butler GS, Jamroz A, Azizi $P$, Overall $C M$ and Bromme D (2018) Aging-associated modifications of collagen affect its degradation by matrix metalloproteinases. Matrix Biol 65, 30-44

3. Fanjul-Fernandez $M$, Folgueras $A R$, Cabrera $S$ and Lopez-Otin C (2010) Matrix metalloproteinases: evolution, gene regulation and functional analysis in mouse models. Biochim Biophys Acta 1803, 3-19

4. Page-McCaw A, Ewald AJ and Werb Z (2007) Matrix metalloproteinases and the regulation of tissue remodelling. Nat Rev Mol Cell Biol 8, 221-233

5. Ala-aho R and Kahari VM (2005) Collagenases in cancer. Biochimie 87, 273-286

6. Bashir MM, Sharma MR and Werth VP (2009) TNF-alpha production in the skin. Arch Dermatol Res 301, 87-91

7. Gashler A and Sukhatme VP (1995) Early growth response protein 1 (Egr-1): prototype of a zinc-finger family of transcription factors. Prog Nucleic Acid Res Mol Biol 50, 191-224

8. Shin SY, Lee JM, Lim Y and Lee YH (2013) Transcriptional regulation of the growth-regulated oncogene alpha gene by early growth response protein- 1 in response to tumor necrosis factor alpha stimulation. Biochim Biophys Acta 1829, 1066-1074

9. Grimbacher B, Aicher WK, Peter HH and Eibel H (1998) TNF-alpha induces the transcription factor Egr-1, proinflammatory cytokines and cell proliferation in human skin fibroblasts and synovial lining cells. Rheumatol Int 17, 185-192

10. Okada M, Fujita T, Sakaguchi T et al (2001) Extinguishing Egr-1-dependent inflammatory and thrombotic cascades after lung transplantation. FASEB J 15, 2757-2759

11. Ji B, Chen XQ, Misek DE et al (2003) Pancreatic gene expression during the initiation of acute pancreatitis: identification of EGR-1 as a key regulator. Physiol Genomics 14, 59-72

12. Ko SW, Vadakkan $\mathrm{KI}$, Ao $\mathrm{H}$ et al (2005) Selective contribution of Egr1 (zif/268) to persistent inflammatory pain. J Pain 6, 12-20

13. Cho SJ, Kang MJ, Homer RJ et al (2006) Role of early growth response-1 (Egr-1) in interleukin-13-induced inflammation and remodeling. J Biol Chem 281, 8161-8168

14. Son SW, Min BW, Lim Y, Lee YH and Shin SY (2008) Regulatory mechanism of TNFalpha autoregulation in HaCaT cells: the role of the transcription factor EGR-1. Biochem Biophys Res Commun 374, 777-782

15. Jeong SH, Kim HJ, Jang $Y$ et al (2014) Egr-1 is a key regulator of IL-17A-induced psoriasin upregulation in psoriasis. Exp Dermatol 23, 890-895

16. Ryu WI, Lee H, Kim JH, Bae HC, Ryu HJ and Son SW (2015) IL-33 induces Egr-1-dependent TSLP expression via the MAPK pathways in human keratinocytes. Exp Dermatol 24, 857-863

17. Shin SY, Kim JH, Baker A, Lim Y and Lee YH (2010) Transcription factor Egr-1 is essential for maximal matrix metalloproteinase- 9 transcription by tumor necrosis factor alpha. Mol Cancer Res 8, 507-519

18. Kim J, Jung E, Choi J, Min DY, Lee YH and Shin SY (2019) Leptin is a direct transcriptional target of EGR1 in human breast cancer cells. Mol Biol Rep 46, 317-324

19. Sun $Y$, Wenger L, Brinckerhoff CE, Misra RR and Cheung HS (2002) Basic calcium phosphate crystals induce matrix metalloproteinase-1 through the Ras/mitogen-activated protein kinase/c-Fos/AP-1/metalloproteinase 1 pathway. Involvement of transcription factor binding sites AP-1 and PEA-3. J Biol Chem 277, 1544-1552

20. Westermarck J, Seth A and Kahari VM (1997) Differential regulation of interstitial collagenase (MMP-1) gene expression by ETS transcription factors. Oncogene 14, 2651-2660

21. Nakashima A, Ota A and Sabban EL (2003) Interactions between Egr1 and AP1 factors in regulation of tyrosine hydroxylase transcription. Brain Res Mol Brain Res 112, 61-69

22. Utreras E, Futatsugi A, Rudrabhatla P et al (2009) Tumor necrosis factor-alpha regulates cyclin-dependent kinase 5 activity during pain signaling through transcriptional activation of p35. J Biol Chem 284, 2275-2284

23. Mishra JP, Mishra S, Gee K and Kumar A (2005) Differential involvement of calmodulin-dependent protein kinase Il-activated AP-1 and c-Jun N-terminal kinaseactivated EGR-1 signaling pathways in tumor necrosis factor-alpha and lipopolysaccharide-induced CD44 expression in human monocytic cells. J Biol Chem 280, 26825-26837

24. Son SW, Min BW, Lim Y, Lee YH and Shin SY (2008) Regulatory mechanism of TNFalpha autoregulation in HaCaT cells: the role of the transcription factor EGR-1. Biochem Biophys Res Commun 374, 777-782

25. Segawa R, Shigeeda K, Hatayama T et al (2018) EGFR transactivation is involved in TNF-alpha-induced expression of thymic stromal lymphopoietin in human keratinocyte cell line. J Dermatol Sci 89, 290-298

26. Kim SH, Yu HS, Park HG et al (2013) Egr1 regulates lithium-induced transcription of the Period 2 (PER2) gene. Biochim Biophys Acta 1832, 1969-1979

27. Min DY, Jung E, Kim J, Lee YH and Shin SY (2019) Leptin stimulates IGF-1 transcription by activating AP-1 in human breast cancer cells. BMB Rep 52, 385-390 